\title{
A Patient with Acute Liver Failure Due to Acute Hepatitis A
}

\author{
Ike Rahayu Widuri, Iswan Abbas Nusi, Poernomo Boedi Setiawan, Herry Purbayu, Titong \\ Sugihartono, Ummi Maimunah, Ulfa Kholili, Budi Widodo, Muhammad Miftahussurur, Husin \\ Thamrin and Amie Vidyani \\ Department of Internal Medicine, Faculty of Medicine, Universitas Airlangga, Dr. Soetomo General Hospital, Jl. Prof dr. \\ Moestopo 47 Surabaya 60132, Indonesia \\ apji@fk.unair.ac.id
}

Keywords: Acute liver failure, BCAA, HAV, HBV, INR.

Abstract: A 21-year-old male patient was admitted to the Emergency room of Dr. Soetomo General Hospital with a chief complaint of decrease in consciousness. Jaundice and mental status changes were found. Laboratory testing showed hyperbilirubinemia, progressive rise in serum alanine and aspirate aminotransferase levels, rise in prothrombin time and International Normalized Ratio (INR), positive IgM anti HAV. Diagnosis of this patient was acute liver failure due to acute hepatitis A. The patient was treated treatment with $\mathrm{O}_{2}$ nasal 3-4 lpm, a low-protein diet, fluid therapy including $\mathrm{NaCl} 0.9 \%$, dextrose $10 \%$ and Branch Chain Amino Acids (BCAA), inj. ranitidine 2x1 amp, vitamin K 3x1 amp, FFP transfusion $10 \mathrm{cc} / \mathrm{kg}$ (first hour) followed by $1 \mathrm{cc} /$ hour, lactulose syrup $4 \times 30 \mathrm{cc}$, L-ornithine-L-aspartate 4 ampoule/PZ $100 \mathrm{cc}$ for 4 hours. The patient was discharged in good condition. Three weeks later, the patient was monitored at the outpatient clinic, and jaundice was not found.

\section{INTRODUCTION}

Acute liver failure (ALF) refers to the development of severe acute liver injury with encephalopathy and impaired synthetic function (INR of $\geq 1.5$ ) in a patient without cirrhosis or preexisting liver disease. While the time course that differentiates ALF from chronic liver failure varies between reports, a commonly used cutoff is illness duration of $<26$ weeks. ALF can be subcategorized based on how long the patient has been ill and various cutoffs have been used. ALF is classified as hyper acute $(<7$ days), acute ( 7 to 21 days), or subacute ( $>21$ days and $<26$ weeks). ALF has also been referred to as fulminant hepatic failure, acute hepatic necrosis, fulminant hepatic necrosis, and fulminant hepatitis. Untreated, the prognosis is poor; thus, the recognition and management of patients with ALF is crucial (Chopra, 2015).

Hepatitis A is a disease of the liver caused by the hepatitis A virus (HAV). HAV is a single-stranded RNA virus, a member of the Hepatovirus genus of the Picornaviridae family. HAV causes an acute self-limiting illness, usually spread through the fecal-oral route of transmission. Cases occur most frequently among children and young adults. Rarely, a person may develop fulminant hepatitis A, which is characterized by severe symptoms and may be fatal (Cheney, 2015).

The incidence of ALF in developed countries is between one and six cases per million people per year. The incidence rate may be higher in developing countries, but the data are lacking. The etiology of ALF varies demographically (Panackel, 2015). In Australia, Denmark, the United Kingdom, and the United States, acetaminophen is the most common cause of ALF, whereas in Asia and some other parts of Europe, viral hepatitis predominates (Chopra, 2015).

Hepatotropic viruses are the most common cause of ALF in developing countries. ALF occurs in $<1 \%$ of cases of acute hepatitis A (Panackel, 2015). The majority of acute hepatitis A infections do not result in ALF, and many young patients with acute hepatitis A are anicteric and asymptomatic (Torres, 2010). Although ALF due to hepatitis A is very rare, it needs a deeper understanding; thus, we report a case of a young male with ALF caused by acute hepatitis A. 


\section{CASE}

A male, Mr. MR, 21 years old, single, Moslem, Makassarese, lives in Surabaya, currently receiving education in "Akademi Penerbangan" (patient was staying in a dormitory during his education), was referred by Bhayangkara Hospital to the Emergency room of Dr. Soetomo General Hospital on August 13, 2015 with a chief complaint of decrease in consciousness.

The patient's consciousness had decreased, starting with confusion 12 hours before he was referred to Dr. Soetomo General Hospital. Previously, his vision was blurry one day before he was referred to Dr. Soetomo General Hospital and he lost his sight a few hours later. The patient had been feverish for 5 days, his eyes turned yellow for 3 days, he felt nausea with vomiting and abdominal discomfort, plus loss of appetite $(+)$. He had noticed that his urine was much darker than normal. There was no complaint with defecation. The patient underwent a medical examination in Bhayangkara Hospital and was hospitalized there for 3 days. Bhayangkara Hospital diagnosed him with hepatitis A. The patient was then referred to Dr. Soetomo General Hospital because his condition continuously declined and he lost his sight.

$\mathrm{He}$ had no history of diabetes mellitus, hypertension, smoking, drug abuse, or liver disease. The family history of diabetes mellitus and hypertension was not found. No other family members had a similar disease. His general condition was weak, the patient was confused with a GCS of 3-4-5, blood pressure of $160 / 96 \mathrm{mmHg}$, regular pulses of $90 \mathrm{bpm}$, respiration of $22 \mathrm{bpm}$ and axillary temperature of $37.5^{\circ} \mathrm{C}$. Examination of the head and neck showed jaundice, no anemia, cyanosis, and dyspnea and no lymph node enlargement. Examination of the thorax found symmetrical breathing movements, right and left vesicular breath sounds, and no rhonchi or wheezing. On cardiac examination, the apex beat was palpated in the $5^{\text {th }}$ intercostal space of the left midclavicular line, S1 and S2 were single and no murmurs, with no gallops or extra-systole. On abdominal examination, normal positive bowel sounds were found, the liver was palpable one finger below the right costal arch and the spleen was not palpable, plus tenderness was found on right upper abdominal quadrant. On extremity examination, the acral areas were warm, dry red, and there was no leg edema.

Laboratory examination (August $13^{\text {th }}$ 2015): Hb $14.3 \mathrm{~g} / \mathrm{dL}$, leukocytes $13.560 / \mathrm{uL}$, neutrophils $58.8 \%$, thrombocytes $303,000 / \mathrm{uL}$, HCT $45 \%$, random blood glucose $80 \mathrm{mg} / \mathrm{dL}$, serum creatinine $1.12 \mathrm{mg} / \mathrm{dL}$, BUN $16 \mathrm{mg} / \mathrm{dL}$, direct bilirubin $7.29 \mathrm{mg} / \mathrm{dL}$, total bilirubin $11.43 \mathrm{mg} / \mathrm{dL}$, ALT $1867 \mathrm{IU} / \mathrm{L}$, AST 371 IU/L, albumin $3.4 \mathrm{~g} / \mathrm{dL}$, CRP $1.72 \mathrm{mg} / \mathrm{L}$, potassium $4.17 \mathrm{mmol} / \mathrm{L}$, sodium $132.1 \mathrm{mmol} / \mathrm{L}$, chloride 95.5 mmol/L, HbsAg: non-reactive, PPT 41.7 seconds (control 11), APTT 86.5 seconds (control 26.1), IgM anti HAV (+). Urinalysis: SG 1.015, pH 6.5, prot $(+1)$, glu $(-)$, keton $(-)$, nitrite $(-)$, leukocyte $(-)$, urobilinogen $(+2)$, bilirubin $(+2)$. Blood gas analysis: $\mathrm{pH} 7.53, \mathrm{pCO} 235, \mathrm{pO}_{2} 99, \mathrm{HCO}_{3} 29.2$, Beecf 6.5, $\mathrm{SO}_{2} 98 \%$.

Chest X-ray: cor and pulmo were within normal limits. Abdominal ultrasonography imaging: Hepar: normal size, regular edge, sharp angle, echo parenchyma intensity was homogeneous. Portal vein and hepatic vein appeared normal. IHBD/EHBD had no enlargement. Mass/cyst/abscess (-). Conclusion: no visible abnormalities. Consultation to Ophthalmology Department: oculi right and sinistra: visus: difficult to evaluate. Conclusion: normal fundus. Suggestion: re-consultation if the condition is adequate. Consultation to Neurology Department: signs of neurological deficit: not found, meningeal sign: not found. Based on anamnesis, history of illness, clinical, laboratory and adjunctive examination, the patient was diagnosed with acute liver failure e.c. acute hepatitis A. Planning of diagnosis: anti $\mathrm{HCV}$, serial liver function tests, serial RBG, PPT and APTT. Planning of therapy: $\mathrm{O}_{2}$ nasal 3-4 lpm, EH diet III 1,200 kcal/day (start with sonde 6x100 cc), IVFD NaCl 0.9\%: D10\% : BCAA 1:1:1 $21 \mathrm{dpm}$, inj. cefotaxime $3 \times 1$ gram iv, inj. ranitidine $2 \times 1 \mathrm{amp}$ iv, inj. metoclopramide $3 \times 1 \mathrm{amp}$ iv, inj. vitamin $\mathrm{K} 3 \times 1$ amp, UDCA $3 \times 250 \mathrm{mg}$ p.o., hepatoprotector $3 \times 1$ p.o., lactulose syrup 4x $30 \mathrm{cc}$. Planning of monitoring: complaint and vital sign.

$2^{\text {nd }}$ day of admission: $\mathrm{S}$ : decrease in consciousness, with a GCS of 3-4-5, blurred vision. O: blood pressure $140 / 90 \mathrm{mmHg}$, pulse 82 beats/minutes, respiratory rate $20 \mathrm{x} /$ minutes, temperature $37.0^{\circ} \mathrm{C}$, jaundice. Consultation to Psychiatry Department. Conclusion: the lucid phase of delirium and organic mental disorder. A: acute liver failure e.c. acute hepatitis A. Planning of diagnosis: Consultation to Ophthalmology Department, ALT, AST, direct bilirubin, indirect bilirubin/3 days, serial RBG, PPT and APTT post FFP, rapid diagnostic serological tests (Lepto Tek), procalcitonin, blood and urine culture. Planning of therapy:

The patient's treatments were the same as the day before, with added Fresh Frozen Plasma (FFP) 
transfusion $450 \mathrm{cc} /$ hour followed by $150 \mathrm{cc} / 3$ hours, L-ornithine-L-aspartate 4 ampoule/PZ $100 \mathrm{cc}$ for 4 hours. Planning of monitoring: complaint and vital signs.

$3^{\text {rd }}$ day of admission: S: general condition was weak, with a GCS of 4-5-6, blurred vision. O: blood pressure 150/90 $\mathrm{mmHg}$, pulse 78 beats/minutes, respiratory rate $20 \mathrm{x} /$ minutes, temperature $37.0^{\circ} \mathrm{C}$, jaundice. PPT 31.6 seconds (9-12), APTT 85.0 seconds (23-33), RBG $101 \mathrm{mg} / \mathrm{dl}$, IgG leptospira negative, IgM leptospira negative, anti HCV nonreactive, procalcitonin 2.11. A: acute liver failure e.c. acute hepatitis A. Planning of diagnosis: RBG, PPT and APTT post FFP. Planning of therapy: The patient's treatments were the same as the day before. Planning of monitoring: complaint and vital signs.

$4^{\text {th }}$ day of admission: S: general condition was weak, with a GCS of 4-5-6. The patient underwent improvement, with the blurred vision getting better. O: blood pressure $150 / 90 \mathrm{mmHg}$, pulse 76 beats/minutes, respiratory rate $20 \mathrm{x} /$ minutes, temperature $36.5^{\circ} \mathrm{C}$, jaundice. RBG $98 \mathrm{mg} / \mathrm{dl}$, PPT 22.6 seconds (9-12), APTT 62.9 seconds (23-33), INR 2.06. ALT 555 IU/L, AST 92 IU/L. Consultation to Ophthalmology Department: The ophthalmoscopy examination: fundus reflex $+/+$, retinal bleeding -/-, exudate $-/-$, retinal microaneurysm -/-, macular reflex $+/+$. Conclusion: the retina, blood vessels, and the optic disc appeared normal. Planning of diagnosis: PPT and APTT post FFP. Planning of therapy: $\mathrm{H} 2$ diet 2,100 kcal/day, the patient's treatments were the same as the day before, with added Fresh Frozen Plasma transfusion $450 \mathrm{cc} / 1$ hour followed by $150 \mathrm{cc} / 3$ hours.

$5^{\text {th }}$ day of admission: $S$ : the general condition was adequate; the patient was compos mentis with a GCS of 4-5-6, and blurred vision was getting better. O: blood pressure $140 / 90 \mathrm{mmHg}$, pulse 74 beats/minutes, respiratory rate $20 \mathrm{x} /$ minutes, temperature $36.5^{\circ} \mathrm{C}$, jaundice. RBG $91 \mathrm{mg} / \mathrm{dL}$, PPT 16.5 seconds (9-12), APTT 38.6 seconds (23-33), INR 1.51. Review of ultrasonography imaging with conclusion: hepatomegaly and gallbladder sludge. Planning of diagnosis: toxicology. Planning of therapy: IVFD D10\%: BCAA 2:1 $21 \mathrm{dpm}$. Other therapies continued.

$7^{\text {th }}$ day of admission: S: the general condition was adequate; the patient was compos mentis with a GCS of 4-5-6, and the blurred vision was getting better. O: blood pressure $120 / 80 \mathrm{mmHg}$, pulse 70 beats/minutes, respiratory rate $20 \mathrm{x} /$ minutes, temperature $\quad 36.5^{\circ} \mathrm{C}$, jaundice. Laboratory examination result: $\mathrm{Hb} 14.6 \mathrm{~g} / \mathrm{dL}$, leukocytes 15,090/uL, neutrophils $67.9 \%$, thrombocytes
$305,000 / \mathrm{uL}$, HCT $42 \%$, random blood glucose 87 $\mathrm{mg} / \mathrm{dL}$, serum creatinine $0.5 \mathrm{mg} / \mathrm{dL}$, BUN $16 \mathrm{mg} / \mathrm{dL}$, direct bilirubin $8.7 \mathrm{mg} / \mathrm{dL}$, total bilirubin 13.07 mg/dL, ALT 336 IU/L, AST 113 IU/L, albumin 3.4 $\mathrm{g} / \mathrm{dL}, \quad$ CRP $0.4 \mathrm{mg} / \mathrm{L}$, potasium $4.12 \mathrm{mmol} / \mathrm{L}$, natrium $136 \mathrm{mmol} / \mathrm{L}$, chloride $96 \mathrm{mmol} / \mathrm{L}$, PPT 12.6 seconds (9-12), APTT 38.9 seconds (23-33), INR 1.16 .

Toxicology test: negative. Planning of therapy: the patient's treatments were the same as the day before.

$10^{\text {th }}$ day of admission: $\mathrm{S}$ : the general condition was adequate; the patient was compos mentis with a GCS of 4-5-6. O: blood pressure 120/80 $\mathrm{mmHg}$, pulse 70 beats/minutes, respiratory rate $20 \mathrm{x} /$ minutes, temperature $\quad 36.5^{\circ} \mathrm{C}$, jaundice. Laboratory examination result: $\mathrm{Hb} 13.7 \mathrm{~g} / \mathrm{dL}$, leukocytes $10,020 / \mathrm{uL}$, neutrophils $63.9 \%$, thrombocytes 260,000/uL, HCT 40.3\%, random blood glucose 102 $\mathrm{mg} / \mathrm{dL}$, direct bilirubin $8.98 \mathrm{mg} / \mathrm{dL}$, total bilirubin $14.96 \mathrm{mg} / \mathrm{dL}$, ALT $226 \mathrm{IU} / \mathrm{L}$, AST $98 \mathrm{IU} / \mathrm{L}$, blood and urine culture: sterile. Planning of therapy: the patient's treatments were the same as the day before.

$12^{\text {th }}$ day of admission: No complaint of the condition. Blood pressure $120 / 80 \mathrm{mmHg}$, pulse 82 beats/minutes, respiratory rate $20 \mathrm{x} /$ minutes, temperature $\quad 36.5^{\circ} \mathrm{C}$, jaundice. Laboratory examination result: direct bilirubin $7.14 \mathrm{mg} / \mathrm{dL}$, total bilirubin $11.72 \mathrm{mg} / \mathrm{dL}$, ALT $145 \mathrm{IU} / \mathrm{L}$, AST 91 IU/L. Patient was discharged and suggested to visit Gastroenterohepatology policlinic the next week. The patient was treated with a vitamin $\mathrm{K} 3 \times 100 \mathrm{mcg}$ p.o., UDCA $3 \times 250 \mathrm{mg}$ p.o., hepatoprotector $3 \times 1$ p.o., L-ornithine-L-aspartate $3 \times 2$ sachet.

A week after being discharged, the patient came to the clinic in a better condition, and the laboratory result was: random blood glucose $121 \mathrm{mg} / \mathrm{dL}$, direct bilirubin $5.41 \mathrm{mg} / \mathrm{dL}$, total bilirubin $9.17 \mathrm{mg} / \mathrm{dL}$, ALT $81 \mathrm{IU} / \mathrm{L}$, AST $45 \mathrm{IU} / \mathrm{L}$. Three weeks later, the patient was monitored at the outpatient clinic, and jaundice was not found. The laboratory result: direct bilirubin $0.91 \mathrm{mg} / \mathrm{dL}$, total bilirubin $1.26 \mathrm{mg} / \mathrm{dL}$, ALT 48 IU/L, and AST 36 IU/L.

\section{DISCUSSION}

As mentioned above, ALF occurs on the basis of acute hepatocellular injury caused by toxic, viral or metabolic stress or hypotension. However, regardless of the initial type of liver injury, ALF propels a series of events inducing hepatocellular necrosis and apoptosis, reducing the regeneration capacity of the liver. Massive loss of hepatocytes 
reduces the functional capacity of the liver for glucose, lipid and protein metabolism, biotransformation, and synthesis of coagulation factors, leading to encephalopathy, coagulopathy, hypoglycemia, infections, and renal and multi-organ failure (Altinbas, 2015). The initial clinical presentation of ALF is often nonspecific and easily misdiagnosed, as it often occurs in otherwise healthy individuals without a history of chronic liver disease (Torres, 2010). The clinical presentation of ALF varies, and symptoms can range from mild gastrointestinal confusion to generalized malaise to confusion (Cox, 2009). Malaise, fatigue, nausea and/or vomiting usually appear first, followed by jaundice. Mental status changes usually appear after the onset of jaundice (Torres, 2010). Other clinical manifestations may include hepatomegaly, lethargy, anorexia, pruritus, right upper quadrant tenderness, and abdominal distension from ascites. As the liver failure progresses, patients who were initially anicteric may develop jaundice, and those with subtle mental status changes (e.g. lethargy, difficulty sleeping) may become confused or eventually comatose (Chopra, 2015).

Laboratory test abnormalities are typically seen in patients with acute liver failure including:

Prolonged prothrombin time, resulting in an INR $\geq$ 1.5 (this finding is part of the definition of acute liver failure and thus must be present); elevated aminotransferase levels (often markedly elevated); elevated bilirubin level; low platelet count ( $\leq$ $150,000 / \mathrm{mm} 3)$. Other laboratory findings that can be seen in patients with ALF include: anemia, elevated serum creatinine and blood urea nitrogen, elevated amylase and lipase, hypoglycemia, hypophosphatemia, hypomagnesemia, hypokalemia, acidosis or alkalosis, elevated ammonia level, elevated lactate dehydrogenase (LDH) level (Chopra, 2015). The liver has a central role in the synthesis of most coagulation factors and some inhibitors of coagulation and fibrinolysis. Deficiencies of fibrinogen, factors II, V, VII, IX and $\mathrm{X}$, and platelet are often present in ALF (Munoz, 2010). The synthesis of coagulation factors is universally decreased, while consumption of clotting factors and platelets also may occur; thus, platelet counts frequently drop to $\leq 150,000 / \mathrm{mm} 3(50-70 \%)$ (Lee, 2011).

Hepatic encephalopathy is a common complication in patients with ALF. The altered sensorium that occurs in ALF is believed to be caused by abnormalities in the regulation of neurotransmitters (Cox, 2009). Arterial ammonia levels rise and appear to contribute to astrocyte swelling. Levels greater than 150 to $200 \mathrm{mmol} / \mathrm{L}$ have been shown to correlate with cerebral edema and herniation (Tujios, 2011). Intracranial hypertension from severe cerebral edema remains a feared complication and is a leading cause of death worldwide among patients with ALF. About 50$80 \%$ of ALF patients have renal failure. Druginduced nephrotoxicity; acute tubular necrosis; and abdominal compartment syndrome are the main causes (Panackel, 2015). This complication is more common in the elderly and in patients with acetaminophen-induced ALF. Circulatory dysfunction and hypotension are common in patients with ALF (Bernal, 2013).

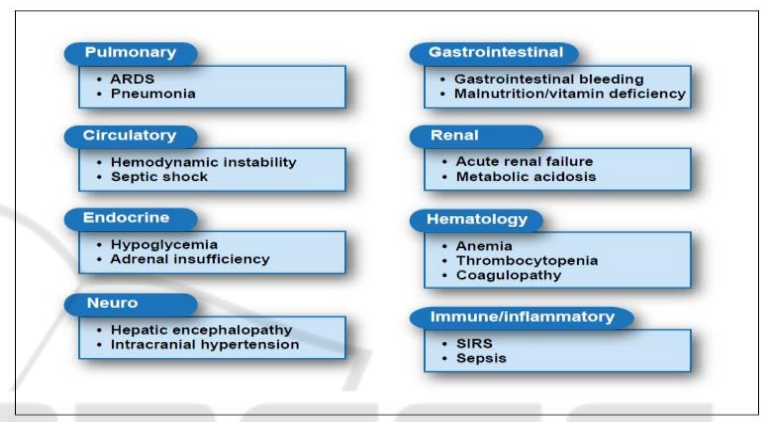

Figure 1: Acute liver failure involvement of multiple organ systems (Torres, 2010).

In this case, the patient was referred to the Emergency room of Dr. Soetomo General Hospital with a chief complaint of decrease in consciousness. There was no impairment of the liver previously. No alcohol and medications were used over the last 6 months. The patient showed mental disorientation or confusion, with clinical features of fever and jaundice. He felt nausea, vomiting and abdominal discomfort. From abdominal examination, the liver was palpable one finger below the right costal arch, and tenderness was found on the right upper abdominal quadrant. Laboratory testing showed elevated aminotransferase and bilirubin levels, prolonged prothrombin time with an INR $\geq 1.5$. Based on anamnesis, physical examination and laboratory findings, the patient was diagnosed with acute liver failure.

Acute liver failure can result from a wide variety of causes. Several viruses have been associated with ALF, including hepatitis A, B, C, D, and E (Chopra, 2015). HAV is the second most common viral cause of ALF after HBV (Ichai, 2008). 
Table 1: Etiologies of Acute Liver Failure (Altinbas, 2015).

\begin{tabular}{lll}
\hline Etiology & Subtype & Investigation \\
\hline Intoxication & Drug & Drug concetrations in serum \\
& Amanita & History \\
& Idiosyncratic drug toxicity & Drug concentrations in serum/eosinophil count \\
\hline Viral hepatitis & HAV & IgM HAV \\
& HBV & HBsAG, IgM anti-core, HBV DNA \\
& HBV/HDV & HBsAG, IgM HDV, HDV RNA \\
& Anti-HCV, HCV RNA \\
& HCV & Anti-HEV, HEV RNA \\
& HEV & \\
\hline Immunologic & GVHD & ANA,LKM, SLA, ASMA, IgG \\
& Bilson's disease & \\
\hline Metabolic & & Urinary copper, ceruloplasmin in serum, slit-lamp \\
& examination \\
& ATdeficiency & Ferrintin in serum, transferrin saturation \\
& Hemochromatosis & \\
\hline Vascular & Budd-Chiari syndrome & Ultrasound (Doppler) \\
& Ischemic & Ultrasound (Doppler), echocardiography (ECO) \\
& Veno-occusive disease & Ultrasound (Doppler) \\
\hline Pregnancy-included & HELLP syndrome & Hematocrit test, peripheral blood smear, platelet \\
& count \\
\hline
\end{tabular}

ANA, anti-nuclear antibody; ASMA, anti-smooth muscle antibody;IgM, immunoglobulin M; IgG, immunoglobulin G; HBsAg, hepatitis B surface antigen

Hepatitis A infection has four clinical phases although these do not occur in all patients. The first stage is an incubation period of 15 to 50 days (mean 28 to 30 days). This stage is asymptomatic, but the infected person may be actively shedding the virus in the stools. The second stage is a pre-icteric period of several days to weeks that may precede the onset of jaundice. This prodromal period is characterized by nonspecific symptoms followed by gastrointestinal symptoms such as anorexia (loss of appetite), nausea, vomiting, abdominal pain, fatigue, malaise, and fever. Other symptoms at this stage may include myalgia (muscle pain), arthralgia (joint pain), coughing, pharyngitis, constipation, diarrhea, pruritus (itchiness), and urticaria (hives). Dark urine caused by elevated bilirubin levels usually occurs prior to the onset of jaundice. In the third stage, yellowing of the skin and eyes of jaundice appears and most symptoms subside, although clinical signs such as hepatomegaly and hepatic tenderness are found in about half of patients. Jaundice usually resolves within a few weeks. The final stage is a convalescent period during which the patient recovers (WHO, 2010). The clinical course of HAV infection varies greatly, ranging from asymptomatic, subclinical infections to cholestatic hepatitis or fulminant liver failure (Pischke, 2015).

The diagnosis of acute HAV infection is made by the detection of anti HAV antibodies in a patient with the typical clinical presentation. Serum IgM anti HAV is the gold standard for the detection of acute illness. The presence of serum IgM anti HAV antibodies in adults without clinical features of viral hepatitis does not necessarily indicate acute infection. Anti HAV is positive at the onset of symptoms, peaks during the acute or early convalescent phase of the disease, and remains positive for approximately four to six months. IgG anti HAV appears early in the convalescent phase of the disease and remains detectable for decades (Cheney, 2015).

In this case, a 21-year old man presented with a 5-day history of fever, nausea, vomiting, upper abdominal discomfort, and loss of appetite. He had noticed that his urine was much darker than normal. On examination, he was jaundiced, the liver was palpable one finger below the right costal arch, and tenderness was found on the right upper abdominal quadrant. IgM anti HAV were positive, with raised liver enzyme levels. HbsAg (-), anti HCV (-). Acute 
hepatitis A was diagnosed and confirmed by anamnesis, physical examination and laboratory findings. Acute hepatitis A is mostly self-limiting. Factors which affect the severity of viral hepatitis A consist of host and viral factors. For the viral factors, genetic variation of $5^{\prime}$ nontranslated region (NTR) is related to the severity of the disease whereas the genotype of HAV has no relation to the severity of acute hepatitis A. The host factors such as underlying chronic liver disease, old age, heavy alcohol drinking, HIV infection, pregnancy, etc. are suggested (Kim, 2010).

Specific treatment is not available for acute hepatitis A virus infection. As in most cases, the infection is self-limiting and is followed by complete recovery without chronic sequelae, and no specific interventions are required. Treatment and management of HAV infection are supportive. Treatment of acute viral hepatitis involves resting, relieving symptoms and maintaining adequate intake of fluids (CDC, 2015). During the acute period, general measures such as a healthy diet, plenty of fluids and adequate rest can help an individual to feel better. Hospitalization is warranted for patients whose nausea and vomiting places them at risk of dehydration. Patients who develop fulminant infection require aggressive supportive therapy and should be transferred to a center capable of performing liver transplantation (Cheney, 2015).

In this case, fluid therapy for this patient was normal saline $(\mathrm{NaCl} 0.9 \%)$, and he was also given supportive therapy including ranitidine and metoclopramide.

The general management consists of intensive care support, treatment of specific etiologies and early detection of candidates for liver transplantation. Special attention should be given to coma care, fluid management, hemodynamics, metabolic parameters, and infection control (Panackel, 2015). Patients with grade I encephalopathy may be managed on a general medical ward, quiet environment and frequent neurologic checks (e.g. every two hours) are performed. If there is progression to grade II encephalopathy, or for patients with grade II, III, or IV encephalopathy, management in an intensive care unit is indicated. Serial laboratory tests are used to follow the course of a patient's liver failure and to monitor the complications. Serum aminotransferases and bilirubin should be monitored daily. More frequent monitoring (three to four times daily) should be performed for coagulation parameters, complete blood counts, metabolic panels, and arterial blood gases. In particular, patients should be monitored and treated for hypoglycemia, hypokalemia, hypomagnesemia, and hypophosphatemia (Chopra, 2015).

Hemodynamic derangements are common in patients with ALF due to low systemic vascular resistance. In addition, patients may have intravascular volume depletion because of decreased oral intake and extravasation of fluid into the extravascular space. As a result, most patients will require fluid resuscitation initially. Patients who are hypotensive should be resuscitated with normal saline. Some patients may not respond adequately to fluid resuscitation and may require vasopressor support. Norepinephrine is often preferred because it is considered as the best augmentation of peripheral organ perfusion with less tachycardia and better preservation of splanchnic blood flow than other agents. Dextrose should be added to crystalloid solutions in patients with hypoglycemia (Chopra, 2015). Early restoration of intravascular volume and systemic perfusion can prevent multiorgan failure (Panackel, 2015). Hypoglycemia and hyperglycemia should be avoided in patients with ALF. Glucose should be monitored every 2-6 hours. If the glucose level is below $100 \mathrm{mg} / \mathrm{dL}, \mathrm{D} 10$ infusion is conducted to maintain a serum glucose level above $100 \mathrm{mg} / \mathrm{dL}$ and less than 140- $180 \mathrm{mg} / \mathrm{dL}$ (Patton, 2012). Branched-chain amino acids (BCAA) are used as a therapeutic nutritional supplement in patients with cirrhosis and hepatic encephalopathy (Altinbas, 2015).

ALF is an immunocompromised state due to dysfunction of monocytes, neutrophils, kupffer cells, and complementary systems. ALF is a state of functional immunosuppression that carries a high risk of sepsis. Most common infections are bacterial pneumonia, urinary tract infection, intravenous catheter-induced sepsis, and spontaneous bacterial peritonitis. Fungal infections occur in 30\% of patients with ALF. Frequent sputum, blood and urine culture should be done to detect infection early. Broad spectrum antibiotics may be administered preemptively in patients with coagulopathy, grade III or IV encephalopathy or multiorgan failure (Panackel, 2015). If the decision is made to provide empiric broad spectrum antibiotic coverage, nephrotoxic antibiotics, particularly aminoglycosides, and hepatotoxic antibiotics should be avoided (Chopra, 2015).

Nutritional support is a vital component in the treatment of ALF and should be initiated early. It is required to prevent catabolism of body stores of proteins and it may decrease the risk of gastrointestinal bleeding from stress ulceration in 
critically ill patients. To prevent protein catabolism, severe protein restrictions should be avoided; a daily intake of 60 grams of protein is reasonable for most patients with ALF (Chopra, 2015). Protein restriction is not indicated, and $1 \mathrm{~g} / \mathrm{kg}$ per day of protein is recommended (Torres, 2010). In patients with grade I or II encephalopathy, oral or enteral feeding is usually sufficient to meet metabolic requirements. Enteral feeding should be provided for patients with grade III or IV encephalopathy. If adequate enteral feeding cannot be provided, parenteral nutrition should be initiated (Chopra, 2015).

Ammonia-lowering therapy with lactulose has been the mainstay of treatment for HE (Torres, 2010). Lactulose should be administered at a dose of $30-60 \mathrm{~mL}$ given orally or via a nasogastric tube every 2-6 hours. In patients without good response to oral lactulose or with clinical concerns of ileus, an alternative approach is to administer lactulose via a $150-\mathrm{mL}$ enema plus $350 \mathrm{~mL}$ of tap water every $6-8$ hours (Patton, 2012). Cerebral edema and ICH are the most dramatic complications of ALF. Mannitol therapy $(0.5-1 \mathrm{~g} / \mathrm{kg})$ has been shown to improve survival. Recommended management includes intubation, sedation, and head-of-bed elevation to at least 30 degrees to prevent aspiration pneumonia. Sedation with Propofol is preferred as it may lower intracranial pressure (Tujios, 2011). Head elevation, induction of hypothermia and hyperventilation are recommended by some experts in patients with increased ICP. With worsening brain edema, patients present with systemic hypertension and bradycardia (Cushing reflex), dilated and fixed pupils, and in the end respiratory arrest. Clinical signs of elevated ICP (systemic hypertension, bradycardia and irregular respirations-Cushing's triad) are not uniformly present, and other neurological changes such as pupillary dilatation or signs of decerebration are typically evident only late in the course. L-ornithine L-aspartate (LOLA) reduces ammonia levels by increasing hepatic ammonia disposal and its peripheral metabolism (Altinbas, 2015).

Clinically significant spontaneous bleeding occurs in approximately $5 \%$ of ALF patients. Coagulopathy is managed by routine intravenous or subcutaneous vitamin K. Fresh frozen plasma, cryoprecipitate and platelets are given for INR at or above 1.5, fibrinogen less than $100 \mathrm{mg} / \mathrm{dL}$ and platelets less than $50,000 / \mathrm{mm} 3$ but only if there is active bleeding or prior to invasive procedures (Tujios, 2011). Stress ulcer prophylaxis with an $\mathrm{H} 2$ blocker or proton pump inhibitor is to be given to all patients with ALF, because the most common site of bleeding is the gastrointestinal tract (Panackel, 2015). In cholestatic diseases, the retention of toxic bile acids leads to cell injury. The UDCA counters this effect by stimulating hepatocytes and bile duct epithelial cells to secrete bile. The UDCA at 13-15 $\mathrm{mg} / \mathrm{kg} /$ day is approved for treatment of primary biliary cirrhosis by the United States Food and Drug Administration, and UDCA is the only diseasemodifying agent recommended by the American Association for the Study of Liver Disease (AASLD) for primary biliary cirrhosis. UDCA has antioxidative, cytoprotective and anti-apoptotic effects by reducing cytolysis of hepatocytes. Furthermore, UDCA alter the bile acid pool, thus altering the balance between toxic hydrophobic and non-toxic bile salts (Beuers, 2015).

In this case, therapy for this patient included $\mathrm{O}_{2}$ nasal $3 \mathrm{lpm}$, and $\mathrm{EH}$ diet II 1,400 kcal/day. On the fourth day of treatment, after the patient was conscious, diet was converted to a $\mathrm{H} 2$ diet 2,100 $\mathrm{kcal} /$ day. The patient was also given normal saline $(\mathrm{NaCl}$ 0,9\%), dextrose $10 \%$ and branched-chain amino acids (BCAA), inj. cefotaxime $3 \times 1$ gram iv, inj. ranitidine $2 \times 1$ amp iv, inj. metoclopramide $3 \times 1$ amp iv, inj. vitamin K $3 \times 1$ amp, UDCA $3 \times 250 \mathrm{mg}$ p.o., hepatoprotector $3 \times 1$ p.o., lactulosa syrup $4 \times 30$ cc, fresh frozen plasma (FFP) transfusion $450 \mathrm{cc} / 1$ hour followed by $150 \mathrm{cc} / 3$ hours, L-ornithine-Laspartate 4 ampoule/PZ $100 \mathrm{cc}$ for 4 hours. Complete blood count, serum aminotransferases and bilirubin were monitored every 3 days.

Overall, survival rates in patients treated for ALF are greater than $60 \%$. Approximately $40 \%$ of patients will survive without needing a liver transplantation. The prognosis of patients who are listed for transplantation is variable and cannot always be predicted accurately. Following liver transplantation, the one-year survival rate is approximately $80 \%$. The majority of deaths among patients who encounter liver transplantation are due to neurologic complications or sepsis which occur within three months of the transplantation (Chopra, 2015). Multiple prognostic models have been proposed to help determine the likelihood of spontaneous survival. The most widely applied prognostic system is the King's College Hospital criteria ( $\mathrm{KCH}$ Criteria). These criteria incorporate both the etiology of ALF and clinical parameters of disease (Lee, 2011). 
Table 2: King's College Criteria.

\begin{tabular}{cl}
\hline Acetaminophen-induced ALF \\
\hline Arterial $\mathrm{pH}<7.3$ (regardless of HE) \\
OR all 3 of the following \\
$-\quad$ INR $>6.5$ \\
$-\quad$ Creatine $>300 \mu \mathrm{mol} / 1$ \\
$-\quad$ He grade $3-4$ \\
\hline Non-Acetaminophen-induced ALF \\
\hline INR $>6.5$ (regardless of HE) \\
OR all 3 of 5 of the following (regardless of HE) \\
$-\quad$ Age $<10$ or $>40$ years \\
$-\quad$ Etiology: indeterminated, drug-induced \\
$-\quad$ Time interval icterus to encephalopathy \\
$\quad \quad>7$ days \\
$-\quad$ INR $>3.5$ \\
$\quad$ Bilirubin $>3000 \mu$ mol/1
\end{tabular}

Approximately $\quad 85 \%$ of $\mathrm{HAV}$-infected individuals have full clinical and biochemical recovery within three months and nearly all have recovered in six months. Serum aminotransferase concentrations decrease more rapidly than the serum bilirubin. The latter normalizes in more than $85 \%$ of individuals by three months. Although prognosis of acute hepatitis A is generally favorable, a minority of patients are accompanied by fatal complications (Cheney, 2015).

In this case, the patient was discharged from the hospital in a better clinical condition, even though still having jaundice, but the trend demonstrated decreasing bilirubin. Treatment was continued with vitamin K 3x100 mcg p.o., UDCA 3x250 mg p.o., hepatoprotector $3 \times 1$ p.o., Lornithine-L-aspartate $3 \times 2$ sachet. The patient was suggested to rest at home. The patient was also requested to visit the Gastroenterohepatology policlinic timely.

\section{SUMMARY}

Diagnosis of this patient was acute liver failure due to acute hepatitis $A$. The patient was treated with $\mathrm{O}_{2}$ nasal 3-4 lpm, a low-protein diet, fluid therapy including $\mathrm{NaCl} 0.9 \%$, dextrose $10 \%$ and Branch Chain Amino Acids (BCAA), inj. ranitidine 2x1 amp, vitamin $\mathrm{K} 3 \times 1$ amp, FFP transfusion $10 \mathrm{cc} / \mathrm{kg}$ (first hour) followed by $1 \mathrm{cc} /$ hour, lactulose syrup $4 \times 30$ cc, L-ornithine-L-aspartate 4 ampoule/PZ 100 cc for 4 hours.

\section{REFERENCES}

ALTINBAS, A., BECHMANN, L.P., AKKIZ, H., GERKEN, G., CANBAY, A. 2015. Acute Liver Failure. In : Hepatology - A Clinical Textbook.

BERNAL, W., WENDON, J. 2013. Acute Liver Failure. The New England Journal of Medicine, 369, 2525-34

BEUERS, U., TRAUNER, M., JANSEN, P., POUPON, R. 2015. New Paradigms in The Treatment of Hepatic Cholestasis : From UDCA to FXR, PXR and Beyond. Journal of Hepatology, 62, S25- S37

CDC 2015. Hepatitis A.

CHENEY, C. P. 2015. Overview of hepatitis A virus infection in adults.

CHOPRA, S., GOLDBERG, E. 2015. Acute Liver Failure in Adults: Management and Prognosis.

COX, N. R., MOHANTY, S.R. 2009. Acute Liver Failure. Hospital Physician July/August. 7-15.

ICHAI, P., SAMUEL, D. 2008. Etiology and Prognosis of Fulminant Hepatitis in Adults. Liver Transplantation, 14, S67-S79.

KIM, J. I., KIM, Y.S., JUNG, Y.K., KWON, O.S., KIM, Y.S., KU, Y.S., CHOI, D.J., KIM, J.H. 2010. Factors Influencing the Severity of Acute Viral Hepatitis A. The Korean Journal of Hepatology, 16, 295-300

LEE, W. M., LARSON, A.M., STRAVITZ, R.T. 2011. AASLD Position Paper: The Management of Acute Liver Failure: Update 2011. 1-88

MUNOZ, S. J., PATHIKONDA, M. 2010. Acute Liver Failure. Annals of Hepatology, 9, 7-14

PANACKEL, C., THOMAS, R., SEBASTIAN, B., MATHAI, S.K. 2015. Recent Advances in Management of Acute Liver Failure. Indian Journal of Critical Care Medicine, 19, 27-33.

PATTON, H., MISEL, M., GISH, R.G. 2012. Acute Liver Failure in Adults : An Evidence-Based Management Protocol for Clinicians. Gastroenterology \& Hepatology, 8, 161-174.

PISCHKE, S., WEDEMEYER, H. 2015. Hepatitis A.

TORRES, D. M., STEVENS, R.D., GURAKAR, A. 2010. Acute Liver Failure: A Management Challenge for the Practicing Gastroenterologist. Gastroenterology \& Hepatology, 6, 444-451

TUJIOS, S. R., LEE, W.M. 2011. Acute liver failure. In : Disease of The Liver and Biliary System, Blackwell Publishing. 